\title{
Probing the Origin of Microcracks in Layered Oxide Cathodes via Electron Microscopy
}

\author{
Xiaoming Liu, ${ }^{1, *}$ Xiaowen Zhan, ${ }^{2}$ Zachary D. Hood, ${ }^{3}$ and Miaofang Chi ${ }^{1}$ \\ 1. Center for Nanophase Materials Sciences, Oak Ridge National Laboratory, Oak Ridge, TN USA. \\ 2. Dept. of Chemical and Materials Engineering, University of Kentucky, Lexington, KY USA. \\ 3. Dept. of Materials Science and Engineering, Massachusetts Institute of Technology, Cambridge, \\ MA USA. \\ *Corresponding author: liux1@ornl.gov
}

Nickel-rich layered oxides have been widely used in commercial Li-ion batteries (LIB) due to their high capacity $\left(\sim 200 \mathrm{mAh} / \mathrm{g}\right.$ until $\sim 4.6 \mathrm{~V} v \mathrm{~s}$. $\left.\mathrm{Li} / \mathrm{Li}^{+}\right)$and excellent rate performance. Among this family, $\mathrm{LiNi}_{0.8} \mathrm{Co}_{0.15} \mathrm{Al}_{0.05} \mathrm{O}_{2} \quad(\mathrm{NCA})$ and $\mathrm{LiNi}_{1-\mathrm{x}-\mathrm{y}} \mathrm{Co}_{\mathrm{x}} \mathrm{Mn}_{\mathrm{y}} \mathrm{O}_{2} \quad(\mathrm{NCM}, \quad 1-\mathrm{x}-\mathrm{y} \geq 0.5)$ are two representative layered oxide materials that have attracted the most attention; however, NCA experiences significant capacity fade during long-term cycling [1]. Even though NCM possesses improved energy density and better thermal stability compared to NCA, the performance deterioration is still a critical challenge for LIBs. Thus, understanding the underlying mechanisms of the performance degradation is essential for developing state-of-the-art LIBs, which are suitable for applications in electric vehicles and grid energy storage systems.

During the past decade, extensive efforts have been aimed at revealing the origin of cell performance degradation. It is recognized that Ni-based layered oxide cathodes experience structural, chemical, and mechanical degradation during cycling, for which several mechanisms have been proposed: 1) surface reconstruction of the cathode materials forming a resistive $\mathrm{NiO}$ rock-salt phase; 2) transition metal dissolution by HF attack from the electrolyte; 3) electrolyte oxidation induced by delithiated cathode materials; and 4) microcrack generation within the cathode secondary particles [2-4]. All of these correlated processes result in irreversible changes of the surface and bulk properties of the cathode materials. Our previous work illustrated that by comparing the chemical/structural evolution at the surface, NCA presents better stability than NCM [5] but NCA undergoes severe mechanical degradation. Cracking is generally associated with anisotropic lattice changes during lithiation and delithiation of the cathode materials. Such cracks cause a loss of electrical contact and induce side reactions. While a mechanical understanding of microcracks is well accepted, the role of the electrolyte has been overlooked.

Advanced electron microscopy is a powerful tool to investigate the chemical and structural information at high spatial and energy resolution. In this work, by correlating scanning transmission electron microscopy (STEM) and electron energy loss spectroscopy (EELS), the local structural and chemical evolution of NCA cathode materials at the surface, at grain boundaries, and at microcracks were tracked before and after 1500 cycles, which was challenging for more conventional bulk measurement methods, e.g., X-ray absorption spectroscopy and X-ray diffraction [6]. Our results highlight the impact of the electrolyte on microcrack and void generation and provide new insight for the role of the electrolyte on capacity fading. We found that microcracks initiated and grew along grain boundaries, which also served as pathways for electrolyte penetration into secondary NCA particles. Specifically, voids formed in the center of the cathode secondary particles during cycling, which linked corrosion by the electrolyte and anisotropic lattice strain during lithiation/delithiation that facilitates the loss of electronic contact between NCA particles, ultimately resulting in capacity fading. Our results will serve to 
fundamentally guide microcrack mitigation strategies and improve the long-term cycling performance of next-generation LIBs [6].

References:

[1] MS Whittingham, Chem. Rev. 104 (2004), p. 4271.

[2] S Muto, et al., J. Electrochem. Soc. 156 (2009), p. A371.

[3] G Cherkashinin, et al., Chem. Mater. 27 (2015), p. 2875.

[4] D Aurbach, Journal of Power Sources 89 (2000), p. 206.

[5] W Li, et al., Advanced Energy Materials 8 (2018), 1703154.

[6] Research sponsored by the U.S. Department of Energy, Office of Science, Office of Basic Energy Sciences, Materials Sciences and Engineering Division. Research conducted as part of a user project at ORNL's Center for Nanophase Materials Sciences, a U.S. DOE Office of Science User Facility.
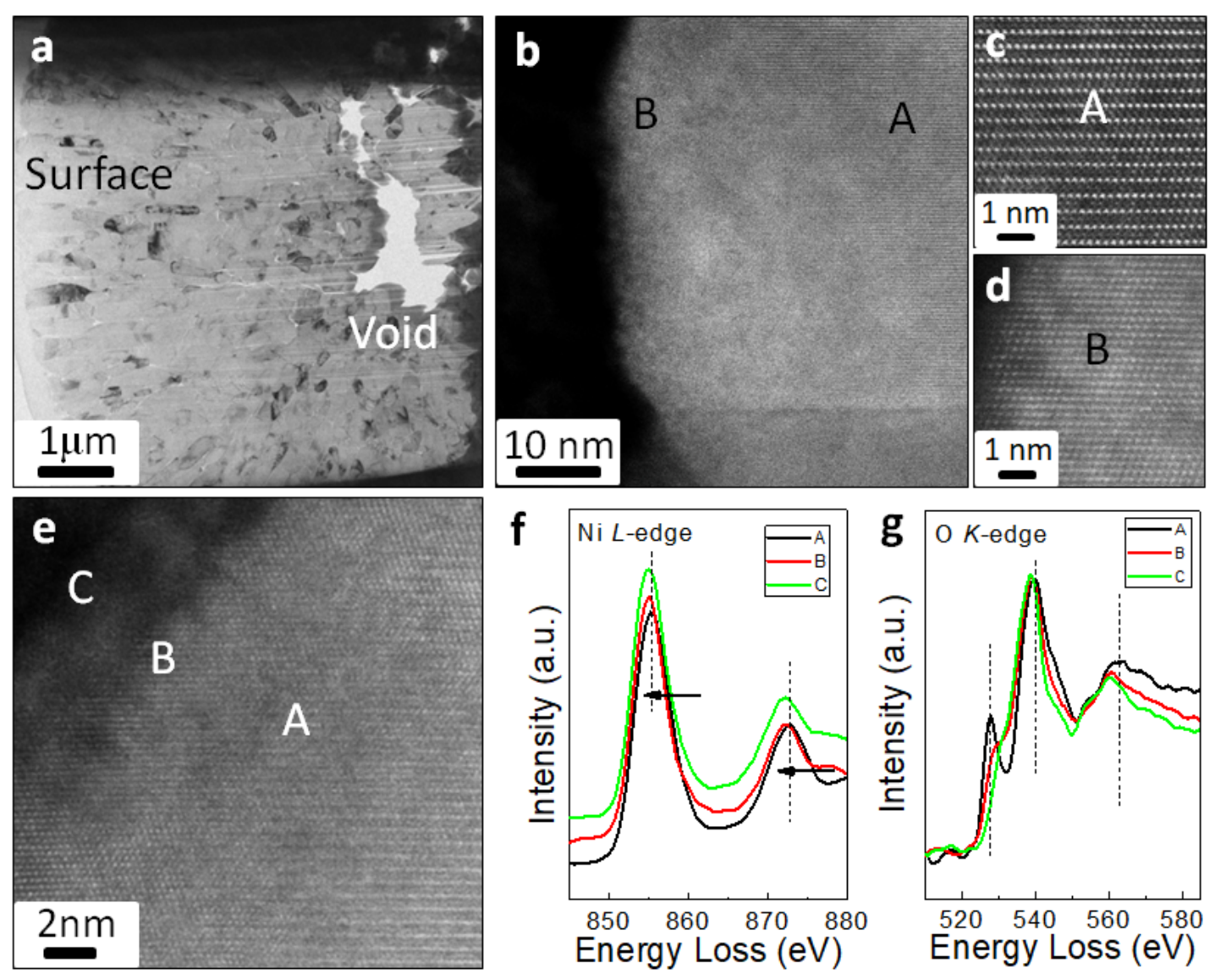

Figure 1. (a) Cross-section TEM image of representative NCA secondary particle after 1500 cycles. High angle annular dark field (HAADF)-STEM images at surface (b) and voids (e) of the secondary NCA particle shown in (a). (c) and (d) are corresponding high-resolution STEM images for regions labeled in (b). [5] Ni $L$-edge (f) and $\mathrm{O} K$-edge (g) EELS spectra for regions labeled in (e). 